\title{
Agility Evaluation of Cloud Agile Enterprise based on AHP and ANN
}

\author{
Miao Ning ${ }^{1}$, Chen Jun ${ }^{2}$, Xu Jing ${ }^{3}$ and Lan Yong ${ }^{1,3}$ \\ Pearl River College of Tianjin University of Finance and Economics, Tianjin, 301800 \\ China University of Mining and Technology, Jiangsu, 221116 \\ Nankai University, Tianjin, 301800 \\ 893962909@qq.com
}

\begin{abstract}
Cloud Agile Enterprise is a cloud computing environment to run the new business model, Agility evaluation is a primary issue in the study of agile enterprise. Cloud computing is based on virtualization technology, take the network as the carrier to provide network infrastructure, platforms, software and other services to form large-scale integration of scalable computing, storage, data, applications and other distributed computing resources for collaborative work of supercomputing mode. As a new model of Internet applications. Cloud computing will become the dominant way that people can access services and information. The meaning of Virtual Enterprise is a temporary union of some independent business process or business. Agility is a key indicator of virtual enterprise, not only passively reflect the company's ability to adapt to market changes, but also suggested that the initiative 's ability to grasp market opportunities, choose to join companies with high agility cloud consisting of virtual enterprise is to improve the basis for virtual enterprise itself. On the basis of the analysis of existing evaluation methods, proposed Comprehensive Analytical Hierarchy Process (AHP) and artificial neural network (ANN) evaluation method. First apply to the Analytic Hierarchy Process (AHP) as ideas to develop evaluation system and then find the right value of evaluation with the AHP. After generating the training sample used to train $A N N$, finally with the trained ANN to evaluate business agility. AHP and ANN results fit are well by simulating, the method can be effectively applied to the evaluation of agility .
\end{abstract}

Keywords: Virtual Enterprises; cloud computing; agility evaluation; AHP; neural network; CLC: TP39 Document Code: A

\section{Introduction}

Cloud computing era, market competition, technological innovation and other aspects of customer needs have taken place enormous changes. The virtual enterprise has been proposed in the cloud computing environment to get new features and content, it is able to adapt to the new environment and with the cloud platform to gain a competitive advantage. Agility is a key indicator of virtual enterprise, because the customer's needs and expectations continue to improve , 
A large-scale single market continue to be segmented, to be differentiation, causing a change in the development of business priorities and competitive strategy. Select companies with high agility joined to form a virtual enterprise, is the foundation of virtual enterprise to improve itself. Agile enterprise adopts a new company way for products and services that was made by customers to make a quick and effective reaction with turn of the market. Flexible organization and coordination of it is actually in information systems, personnel, business and equipment organic combination. Agile companies can offer lower manufacturing costs, increase market share, to meet customer demands, and promote the introduction of new products, reduce non-value added activities and increase the company's competitiveness. Agile Enterprise is the basic unit in the new environment of various production modes - new production models such as Agile Manufacturing, Virtual Enterprise, Agile Supply chain is closely related such as virtual companies can be seen as agile enterprise alliance. So agile enterprise is called business model to adapt to changes and developments in various technologies [1].

\section{Virtual Enterprise Cloud}

Virtual Enterprise is more suitable in a cloud computing environment, stronger and more powerful role can occur .Cloud computing is based on virtualization technology, take the network as the carrier to provide network infrastructure ,platforms, software and other services to form large-scale integration of scalable computing, storage, data, applications and other distributed computing resources for collaborative work of supercomputing mode. Internet applications as a new model. Cloud computing will become the dominant way that people can access services and information. A core concept of cloud computing is to continuously improve the "cloud" processing power, thereby reducing the processing burden on the user terminal, user terminal eventually reduced to a simple input and output devices, and can enjoy the " cloud" computing power on demand ability! Based on the above point of view, we also made a reference definition: "loud computing is based on virtualization technology, take the network as the carrier to provide network infrastructure, platforms, software and other services to form large-scale integration of scalable computing, storage, data, applications and other distributed computing resources for collaborative work, sharing resources, information alliance, virtual enterprise is in the cloud." In the cloud computing model, users no longer need to purchase complex hardware and software, and only need pay the appropriate fees to the "cloud computing" service providers, you can easily get the required computing, storage and other resources over the network. For the definition of special note is needed is an important value of cloud computing on-demand scalability hardware requirements, completely out of the "local" computing, cloud computing data resources just an ideal state, taking into account the private cloud, legacy system reliability, security, and other factors, with the integration of cloud computing resources on-demand extensions of special significance. Agility is a key indicator of virtual enterprise , not only passively reflect the company's ability to adapt to market changes, but also suggested that the 
initiative's ability to grasp market opportunities, choose to join companies with high agility cloud consisting of virtual enterprise is to improve the basis for virtual enterprise itself.

\section{AHP-ANN Evaluation Methods}

To help managers to better enable enterprises to meet the standards agile enterprise , many scholars and experts have a lot of research on the agile enterprise, the first question to be solved is the evaluation of the business agility that enterprise agility in the end how far away from the problem. ChingTorng [2] through the establishment of agility fuzzy inference rules, using fuzzy evaluation method. Van Hoek [3] and others put forward comprehensive evaluation index agility. They define agility index is a comprehensive evaluation of all aspects of agile ability levels. Ren [4] using the Analytic Hierarchy Process (AHP) to evaluate. However, these methods are subjective evaluation method, only gives a subjective evaluation values based on individual preferences. This affects the objectivity of the evaluation results, and the lack of future self-learning, correcting capability.

The artificial neural network (ANN) with self-adapting, self-organizing and self- learning ability, just to make up for the shortcomings of subjective evaluation method. So artificial neural networks was added on the basis of AHP will be used to evaluate. By using AHP will affect the factor of screening, layered, combination, establish evaluation system, and to develop a neural network weight values and formulate input nodes of neural network. Then ANN based on a small number of teachers' information (it is equal to the weight and correlation coefficient, by AHP) training, generated for the evaluation of the neural network to evaluate the agile enterprise. Finally can according to the actual data of agile enterprise operation to achieve the self-learning function of evaluation system by correcting the result and retraining. Both give full consideration to the evaluation of expert experience and intuition thinking mode and reduce the uncertainty of human factors in the evaluation process, both have the advantages of the comprehensive evaluation method and reflects the characteristics of the various evaluation methods.

1) Establish the evaluation system in accordance with AHP thought [5].

2) Calculate the share of each class of each evaluation index weights by AHP.

3) According to the developed index system establish the neural network model.

4) After experts scoring to the enterprise, according to the calculated value of (1), calculated AHP evaluation results.

5) Take the date of (4) as the training samples of neural network, establish the mathematical model of neural network training.

6) Enterprise data input has been trained neural network, obtained evaluation results. Process shown in Figure 1 


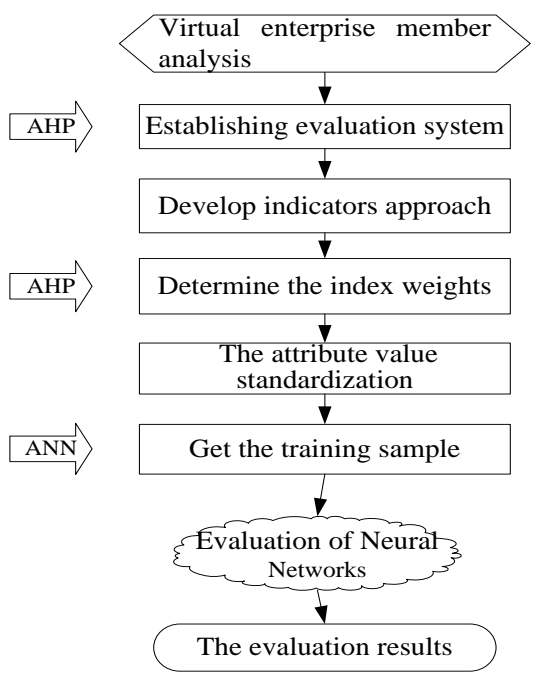

Figure 1. AHP-ANN

\section{Leaguer Agility Evaluation}

The primary task of agile enterprise is to focus the volatile, uncertain and unpredictable factors on business environment, and make the right responses to it. Therefore, agile enterprise needs to have a lot of different agile ability or resilience to survive in the environment. These capabilities constitute agility elements of the agile enterprise [6] . Agility elements proposed by different scholars have some differences, here refer Yusuf [7] and so develop the 32 agility evaluation indicators - -Comprehensive: concurrent execution of multiple activities, mergers, the patency of corporate information to employees. Competition: Business executive or structure is difficult to imitate or copy, multi-directional adventure capability. Team buildings: the team's powers, allowing individuals to form teams, cross-functional teams, cross-enterprise teams. Technology: the sensitivity of new technologies, the leading extent for existing technology, the capabilities to improve the knowledge and skills, flexible manufacturing technologies. Quality: The quality of the product life cycle to ensure that products with high added value, the first of the right decision, short operating cycles. Change: the degree of corporate culture to adapt changes, continuous improvement. Cooperation: the relationships with customers and suppliers. Forming partnerships rapidly, The connection with customers. co-operation with suppliers . Market: the reaction to change market, introducing new plant products, customer-oriented innovation, customer satisfaction rate. Education: Provide ongoing training, the establishment of a learning organization, multi-skilled and flexible staff, staff skills upgrading. Welfare: the satisfaction rate of employee.

Although these 32 indicators are in detail, but seems redundant and messy, most indicators are descriptive, such as "the first time right decision," is more difficult to quantify evaluation . So, in order to use the AHP's idea with it as the foundation to screening, modify indicators and has a reasonable stratification, while according to the new environment of cloud computing to establish a viable evaluation system shown in Figure 2 


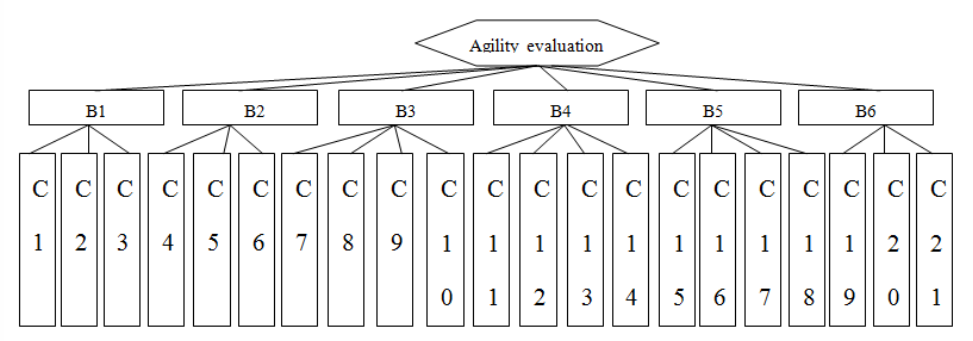

Figure 2. Leaguer Agility Evaluation System

Top A (target layer): the evaluation of the target agility evaluation.

The middle layer $\mathrm{B}$ (criterion level): The evaluation is divided into six agile ability: $\mathrm{B} 1$ : ability to apply technology, B2: staff capacity,B3: Cloud computing management ability, B4: The cloud service performance, B5: The cloud environment competition ability, B6: Ability to work in a cloud environment .The third layer C (factor level): both capability under evaluation factors detailed in each division, a total of 21: C1: New technology sensitivity, C2: Leading technology level, C3: Improve the technical ability, C4: Employee satisfaction, C5: Staff skills upgrading, C6: Decision-making autonomy of employees, C7: Provide training opportunities, C8: Concurrent execution ability, C9: Effect of customer service, C10: Risk ability, C11: The decrease in the number of servers, C12: A quick call to the server, C13: Simple and efficient management, C14: Plan flexibility, C15: The quality of the product, C16: The production cycle, C17: Market growth rate, C18: The added value of the product, C19: Cooperation benefitsC20: Organization reconfiguration ability, C21: Across the enterprise cooperation。

So the paper establishes a clear hierarchy evaluation system, coherent and Factors that layer $\mathrm{C}$ are easy evaluation and comparison. Therefore, use AHP can be a good evaluation, draw weights of each factor.

\section{Factors Indicators Deal}

After the AHP process indicators can generally be attributed to two kinds: efficiency indicators (the bigger the better), cost index (the smaller the better), before use of neural network evaluation expert evaluation is necessary to standardize the evaluation of data processing in its scope interval $[0,1]$, so you can use the following formula

$F_{\mathrm{i}}=\left\{\begin{array}{l}1 ; f_{i} \geq f_{\text {max }} \\ \frac{f_{i}-f_{\text {min }}}{f_{\text {max }}-f_{\text {min }}} ; f_{\text {min }}<f_{i}<f_{\text {max }} \quad \text { efficiency indicators (the bigger the better) } \\ 0 ; f_{i} \leq f_{\text {min }}\end{array}\right.$ 
$F_{i}=\left\{\begin{array}{l}1 ; f_{i} \geq f_{\max } \\ \frac{f_{i}-f_{\min }}{f_{\max }-f_{\min }} \\ 0 ; f_{i} \leq f_{\min }\end{array}\right.$

Which of $F_{\mathrm{i}}$ represents the normalized data one after the other, $f_{i}$ indicating the current condition of the enterprise data, $f_{\min }$ and $f_{\max }$ represent the evaluation criteria used in the optimal face and worst corporate targets.

\section{The Simulation}

In this paper, using Matlab software simulation, neural network and its AHP procedures are written with.

\subsection{AHP Calculate Weights}

In accordance with the above Hierarchical model, the judgment matrix is constructed using pairwise comparison method, employing a number of experts on the evaluation index system in each of the indicators, given the relative scale and calculate the weight of index weight and then plus weights the total average to determine the final assessment index weights. Suppose has $\mathrm{m}$ experts, an indicator $C_{i}(i=1,2 \cdots \sigma)$ for the right of any given weight $K_{i}(i=1,2 \cdots m)$, the final weight of indicator $C_{i}$ is:

$$
W_{i}=\sum_{i=1}^{m} K_{i} / m
$$

Each layer can be obtained relative to the upper right

$$
\begin{aligned}
& W\left(A-B_{t-6}\right)=(0.0368,0.0779,0.1913,0.4247,0.1913,0.0779) \\
& W\left(B_{t}-C_{t-3}\right)=(0.6370,0.1047,0.2583,) \\
& W\left(B_{2}-C_{t-6}\right)=(0.7143,0.1429,0.1429) \\
& W\left(B_{3}-C_{7-t o}\right)=(0.0955,0.0955,0.2495,0.5596) \\
& W\left(B_{4}-C_{t L-t 4}\right)=(0.5222,0.0781,0.1998,0.1998) \\
& W\left(B_{5}-C_{t s-t 8}\right)=(0.3899,0.3899,0.11524,0.0679) \\
& W\left(B_{6}-C_{t 9-2 t}\right)=(0.6000,0.2000,0.2000)
\end{aligned}
$$

By the above judgment matrix of the single sort as a result, the comprehensive factors of target layer calculated the total weight (see Table 1).

Table 1. Total Weights of AHP to Calculate the C Layer

\begin{tabular}{|l|l|l|l|l|l|l|l|l|}
\hline \multirow{2}{*}{} & B1 & B2 & B3 & B4 & B5 & B6 & The total \\
\cline { 2 - 8 } & 0.0368 & 0.0779 & 0.01913 & 0.4247 & 0.1913 & 0.0779 & power of c \\
\hline C1 & 0.6370 & & & & & & 0.0324 \\
\hline
\end{tabular}




\begin{tabular}{|l|l|l|l|l|l|l|l|}
\hline C2 & 0.1047 & & & & & & 0.0038 \\
\hline C3 & 0.2583 & & & & & & 0.0095 \\
\hline C4 & & 0.7143 & & & & & 0.0556 \\
\hline C5 & & 0.1429 & & & & & 0.0111 \\
\hline C6 & & 0.1429 & & & & & 0.0111 \\
\hline C7 & & & 0.0955 & & & & 0.0182 \\
\hline C8 & & & 0.0955 & & & & 0.0182 \\
\hline C9 & & & 0.2495 & & & & 0.0477 \\
\hline C10 & & & 0.5596 & & & & 0.1070 \\
\hline C11 & & & & 0.5222 & & & 0.2217 \\
\hline C12 & & & 0.0781 & & & 0.0331 \\
\hline C13 & & & & 0.1998 & & & 0.0848 \\
\hline C14 & & & 0.1998 & & & 0.0848 \\
\hline C15 & & & & & 0.3899 & & 0.0745 \\
\hline C16 & & & & & 0.3899 & & 0.0745 \\
\hline C17 & & & & & 0.1524 & & 0.0291 \\
\hline C18 & & & & & 0.0679 & & 0.0129 \\
\hline C19 & & & & & & 0.6000 & 0.0467 \\
\hline C22 & & & & & & 0.2000 & 0.0155 \\
\hline C21 & & & & & & 0.2000 & 0.0155 \\
\hline
\end{tabular}

\subsection{Neural Network is Established in this Paper}

Have proved that for any in a continuous function of closed interval can with a single hidden layer feed forward network approximation. So ANN is a choose include input layer, hidden layer and output layer three layers BP network, because the parameters related to the evaluation has 21 , so the number of input layer nodes is 21 as the output layer node number is 1 , on behalf of the member of the virtual enterprise agility. Finally the whole network structure as the 21-15-1 transfer function of hidden layer for tansig, because agile output value between $0 \sim 1$, output layer transfer function for the logsig, learning accuracy of 10-3. Neural network training takes the momentum of the gradient descent method. It introduces a momentum, momentum item refers to the right of each value and the domain change quantity before adding a quantity, so the first $\mathrm{K}$ loops of weights and threshold change can be expressed as

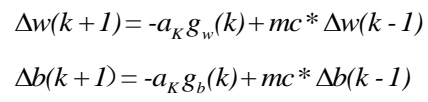

Among them, $g_{w}(k) 、 g_{b}(k)$ respectively represent the performance function of weights and threshold gradient, $a_{K}$ is a vector, $m c$ is the momentum coefficient. Momentum of the gradient descent method has quick training function, and effectively avoids the problem of local minimum BP neural network. 


\subsection{Test and Analysis of the Model}

Select experts to evaluate 12 enterprises, after the data processing and normalized [8], using AHP method to evaluate the results, and then as sample for neural network training, the AHP results comparing with the results of neural network are shown in Table 2.

Table 2. The AHP and ANN Training Results

\begin{tabular}{|l|l|l|l|l|l|}
\hline & AHP & ANN & & AHP & ANN \\
\hline 1 & 0.8598 & 0.8611 & 7 & 0.7544 & 0.7501 \\
\hline 2 & 0.8346 & 0.8317 & 8 & 0.7779 & 0.7808 \\
\hline 3 & 0.8471 & 0.8495 & 9 & 0.7817 & 0.7828 \\
\hline 4 & 0.9046 & 0.9018 & 10 & 0.7373 & 0.7367 \\
\hline 5 & 0.8741 & 0.8646 & 11 & 0.6586 & 0.6584 \\
\hline 6 & 0.8852 & 0.8927 & 12 & 0.6585 & 0.6565 \\
\hline
\end{tabular}

You can see the result of neural network training error is very small (root mean square error less than 3\%). Showed that ANN has a good will of approximation determined by AHP, and then used two not training results to test its ability to predict, according to expert evaluation parameters are shown in Table 9.

Table 3. Prediction Results of AHP and ANN

\begin{tabular}{|l|l|l|}
\hline Enterprise parameters & AHP & ANN \\
\hline 0.910 .930 .930 .920 .990 .960 .920 .96 & 0.8879 & 0.8996 \\
0.950 .940 .960 .930 .930 .980 .920 .92 & & \\
0.920 .720 .940 .840 .85 & & \\
\hline 0.830 .750 .960 .980 .980 .890 .960 .97 & 0.8778 & 0.8841 \\
0.850 .980 .970 .780 .750 .790 .880 .78 & & \\
0.860 .860 .890 .780 .95 & & \\
\hline
\end{tabular}

Results close to, it shows that ANN can independently evaluate the data, and the results are as expected. Afterwards, the actual data of the enterprise can be directly added to the neural network training, thus, the evaluation results of neural network can be more close to reality, which fully reflects the neural network has self- learning ability from the sample.

\section{The Conclusion}

In this paper, the AHP - ANN method was applied to evaluate agility, after establishing the evaluation system, by learning from samples of AHP, fully absorb the summary evaluation expert's experience, knowledge, subjective judgment, and the ability to weigh the importance of targets. Integrated neural network method can be used to better conform to the agile enterprise 
agility evaluation of uncertain nonlinear complex relationship between various factors; and make the evaluation results are objectivity and reliability. You can also self-learning through continuous operation of the enterprise actual data, expand the knowledge capacity, update training of the neural network. However, in a cloud computing environment, virtual enterprise will be more appropriate, and more powerful. Cloud computing as a new computing mode, do not need to purchase or hosting server, do not need to build a data center, but it can take the IT resources, data, application as a service to the user via the Internet; while do not need to recruit IT professionals , cloud computing as a kinds of new infrastructure management, was able to put large、 highly virtualized resource together to form a huge pool of resources, provide unified services. This will make the evaluation results more in line with the actual situation. It is used to evaluate the agility. It can get good results, can provide the necessary technical support and reference for the business transformation into agile enterprises.

\section{References}

[1] A. Abouzied, K. Bajda-Pawlikowski, J. Huang and D. J. Abadi, "HadoopDB inaction: building real word applications [C]", Proceedings of the 2010 international conference on Management of data, New York: ACM, (2010).

[2] M. Slaney and E. Y. Chang, "Processing Web-Scale Multimedia Data [C]", Proceedingsof the international conference on Multimedia, New York: ACM, (2010), pp. 1771-1772.

[3] P. Mika, "Distributed Indexing for Semantic Search [C]", Proceedings of the 3rd International Semantic Search Workshop, New York: ACM, (2010).

[4] IDC. IT Cloud Services User Survey, pt.2: Top Benefits \& Challenges[R/OL], (2008-10-2)[2009-01-21]. http://blogs.idc.com/ie/?p=210.

[5] X. Si Jiang and F. Yan, "Cloud computing is analyzed and the information security [J]", Beijing institute of electronic science and technology, vol. 4, (2008), pp. 1-4.

[6] D. Li Qian and N. Chen, "Extraction of the era of cloud computing GIS development [J]", Anhui agricultural science, vol. 31, no. 72, (2009), pp. 15556-15557155.

[7] J. Geelan, "Twenty One Experts Define Cloud Computing [R/OL]”, (2009-01-04)[2009-02-17].

[8] http://virtualization.sys-con.com/ node/612375.

[9] J. A. Kumar and L. S. Ganesh, "Balancing knowledge strategy: codification and personalization during product development", Journal of Knowledge Management, vol. 15, no. 1, (2011), pp. 118-135. 


\section{Authors}

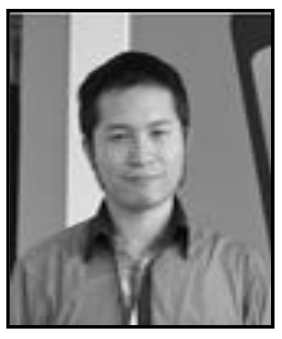

Miao Ning, he received the master's degree in Electrical Engineering from Yanshan University. $\mathrm{He}$ is currently researching on Virtual Enterprise and artificial intelligence.

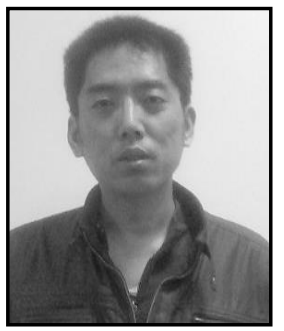

Chen Jun, he received the bachelor's and master's degree in Electrical Engineering from China University of Mining and Technology, and received the doctor degree in Electrical Engineering from China University of Mining and Technology, $\mathrm{He}$ is currently researching on POLSAR image processing, machine learning, the application of remote sensing and image analysis.

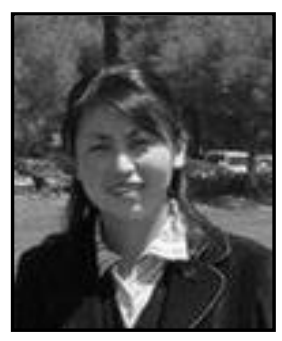

Xu Jing, she got a bachelor's degree in computer science department of nankai university and with a master's degree in Beijing university of aeronautics and astronautics computer department, received a doctor's degree from the department of Nankai University. She mainly engaged in software engineering, software testing, teaching and scientific research work.

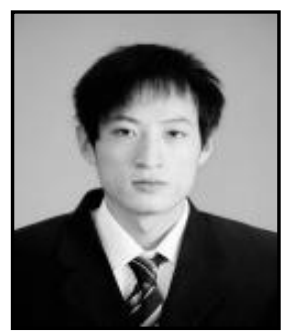

Lan Yong, he got a bachelor's degree in computer science department of Nankai University He is mainly engaged in software engineering. 